\title{
Incidence, risk factors, clinical presentation, and outcomes of hemorrhagic transformation in patients with ischemic stroke admitted to a tertiary hospital in Kingdom of Saudi Arabia
}

Ziad E. Aljundi, MD, Emad O. Miyajan, MBBS, Hassan A. Alharbi, MBBS, Raghad H. Sindi, MBBS, Rawan M. Aldhahwani Medical Student, Zainab N. Bajaber, MBBS, Maher A. Alhazmi, MSc.

\footnotetext{
ABSTRACT

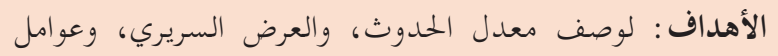

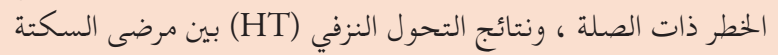

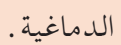

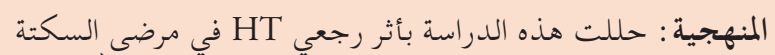

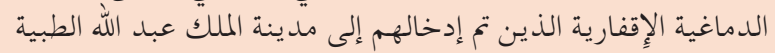

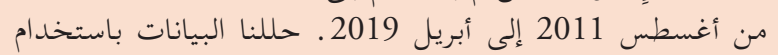

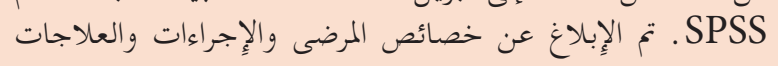
والنتائج · النتائج : كان هناك 504 من مرضى السكتة الدماغية الإِفارية.

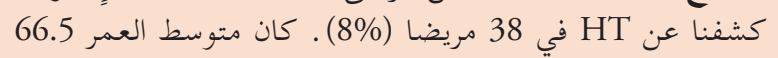

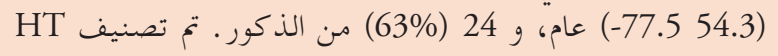

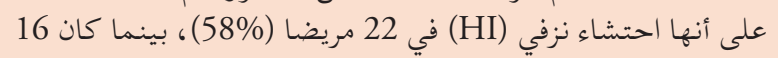

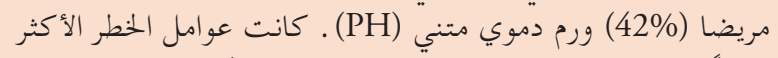

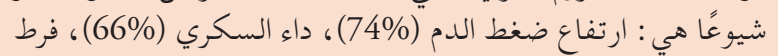

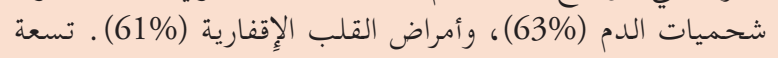

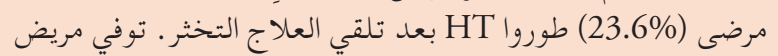

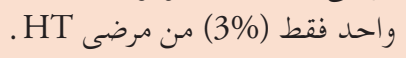

الحلاصة : شكل التحول النزفي (8\%) من جميع مرضى السكتة

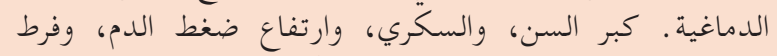

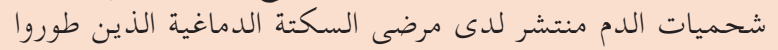

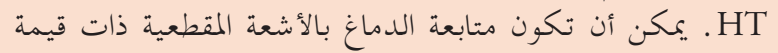

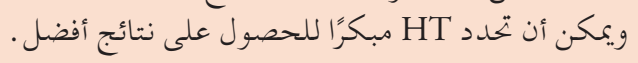

Objectives: To describe the incidence rate, clinical presentation, relevant risk factors, and outcome of hemorrhagic transformation (HT) among ischemic stroke patients.
}

Methods: This retrospective study analyzed HT in patients with ischemic stroke admitted to King Abdullah Medical City from August 2011 to April 2019. Data were analyzed using SPSS. Patients characteristics, procedures, treatments, and outcomes were reported.

Results: There were 504 ischemic stroke patients. The HT was detected in 38 patients ( $8 \%)$. The median age was $66.5(54.3-77.5)$ years, and $24(63 \%)$ were males. HT was classified as hemorrhagic infarction (HI) in 22 patients $(58 \%)$ while 16 patients $(42 \%)$ were parenchymal hematoma $(\mathrm{PH})$. The most common risk factors noted were: hypertension $(74 \%)$, diabetes mellitus (DM) (66\%), hyperlipidemia $(63 \%)$ and ischemic heart disease (IHD) (61\%). Nine patients (23.6\%) developed HT after receiving thrombolytic therapy. Only one patient (3\%) died of HT patients.

Conclusion: HT accounted for (8\%) of all ischemic stroke patients. Old age, DM, hypertension, and hyperlipidemia are frequently encountered with stroke patients who developed HT. Follow up CT brain could be of value and could identify HT early to get better outcomes.

Neurosciences 2020; Vol. 25 (5): 345-349 doi: $10.17712 / n s j .2020 .5 .20200085$

From the Department of Neurology (Aljundi), Executive administration of Research and Innovation (Alhazmi), King Abdullah Medical City, Faculty of Medicine (Miyajan, Alharbi, Sindi, Aldhahwani, Bajaber) Umm Al-Qura University, Makkah, Kingdom of Saudi Arabia

Received 26th May 2020. Accepted 10th July 2020.

Address correspondence and reprint request to: Dr. Ziad E. Aljundi, Department of Neurology, King Abdullah Medical City, Makkah, Kingdom of Saudi Arabia. E-mail: aljundi.ziad@gmail.com ORCID ID: https://orcid.org/0000-0002-7107-714X 
Temorrhagic transformation (HT) which is 1 a spectrum of hemorrhage related to brain ischemia. ${ }^{1}$ The HT is a primary complications of acute ischemic stroke (AIS). ${ }^{2}$ Ischemia makes the cerebral vasculature fragile and more reliable to cause HT after AIS. ${ }^{3-5}$ HT increases both morbidity and mortality rate in the ischemic stroke patients. ${ }^{2}$ The HT affects the outcomes with potential severe short- and longterm effects. ${ }^{6}$ The incidence of HT is between $10-40 \%$ and depends on many factors. ${ }^{1,78}$ Most of HT occurs within 2 weeks of an ischemic stroke. ${ }^{5}$ Previous studies considered old age, hypertension (HTN), diabetes mellitus (DM), large infarct $(>2 \mathrm{~cm})$, reperfusion time, lower platelet count and treatment with thromboticor anticoagulant-therapies as risk factors. ${ }^{9-12}$ The most effective treatment for AIS according to previous studies is recombinant tissue plasminogen activator $(\mathrm{r}-\mathrm{tPA}),{ }^{13}$ however, it appears to increase the risk of symptomatic or asymptomatic HT. ${ }^{1}$ Previous studies also suggest that the outcome of HT patients be strongly associated with HT type hemorrhagic infarction (HI) (Petechiae) or parenchymal hematoma $(\mathrm{PH})$ (a space-occupying lesion), $\mathrm{PH}$ was a significant predictor of both mortality and neurological deterioration. ${ }^{14}$ Thus, it is critical to understand HT and its characteristics especially in our population. This retrospective study was designed to describe the incidence rate, clinical presentation, and relevant risk factors of HT among ischemic stroke patients. Identifying such knowledge could improve the outcome and decrease the risk of HT in AIS patients.

Method. This is a retrospective study conducted in King Abdullah Medical City (KAMC) - a 500bed hospital in Makkah, Kingdom of Saudi Arabia. We identified all patients admitted to KAMC as a case of ischemic stroke from August 2011 to April 2019. Patients who were diagnosed with conditions other than ischemic stroke and did not develop HT were not considered. All patients did a baseline brain computed tomography (CT) scan within 24 hours from symptoms onset and HT detected in follow-up CT imaging reviewed by a stroke neurologist. The HT was defined as 'any degree of hyperdensity within the area of low attenuation. ${ }^{4}$ The definition of small infarct size (lacunar infarct which is lesion $<2 \mathrm{~cm}$ ). ${ }^{15}$ We included

Disclosure. Authors have no conflict of interests, and the work was not supported or funded by any drug company. all the patients who admitted as a case of AIS, and from those we have excluded all the patients who did not developed HT. Of the total ischemic stroke patients, we found 38 patients developed HT (Figure 1). The 38 records were reviewed, and the following data were collected: age, gender, nationality, risk factors, baseline stroke severity scores, laboratory results, radiological reports, vital signs, therapeutic interventions, prescribed medications, complications during hospitalization, stroke severity scores at discharge, length of hospital stay and hospital mortality. The data were collected by 6th-year medical students and supervised by a stroke neurologist.

Data were analyzed using SPSS version 23. Univariable distributions were examined for anomalies, and errors were corrected. Discrete variables were reported using counts and percentages. Continuous variables were examined for normality of distribution visually and by assessing the measure of skewness. Most of the variables were skewed, which indicates that they are not behaving like normal distribution. We also used the Kolmogorov-Smirnov test and Shapiro-Wilk test to test whether the distributions are normal. Almost all the continuous variables have $p$-values that are well below 0.05 . The low $p$-values imply that there is strong evidence that most of the continuous variables are not perfectly normal. Thus, continuous variables were presented as the median and quartiles. The institutional review board (IRB) of King Abdullah Medical City in Makkah approved the study.

Results. A total of 504 ischemic stroke patients admitted to the hospital during the research period. HT was detected in 38 patients accounting for $8 \%$ of all ischemic stroke patients. Patients who developed HT median 66.5 (54.3-77.5) years of age and 24 (63\%) were male. The median National Institutes of Health Stroke Scale (NIHSS) at admission was 10 (5.0-14.2), and the median Glasgow coma scale (GCS) was 14.5 (13.0 - 15.0). Hypertension (73.7\%), DM (65.8\%), hyperlipidemia $(63.2 \%)$, and atrial fibrillation (A-Fib) $(36.8 \%)$ were the most frequent comorbidities. The other comorbidities are listed in (Table 1). The most commonly used medications were antiplatelets (50\%), antihypertensive (86.4\%), and hypoglycemic agents (42.1\%). Regarding stroke etiology, 21 patients (55.3\%) were cardioembolic (Table 1).

A small infarct size $(<2 \mathrm{~cm})$ was found in 5 patients $(13.2 \%)$ and 33 patients $(86.8 \%)$ had medium to large $(>2 \mathrm{~cm})$. The HI occurred in 22 patients $(57.9 \%)$, while $\mathrm{PH}$ was seen in 16 patients $(42.1 \%)$. Thirty-one patients $(81.5 \%)$ had anterior circulation, and 6 patients 
Table 1 - Demographics and baseline characteristics of patients with HT.

\begin{tabular}{|c|c|}
\hline Variables & $\begin{array}{l}\text { Patients with HT } \\
\quad(n=38) n(\%)\end{array}$ \\
\hline \multicolumn{2}{|l|}{ Demographic variables } \\
\hline \multicolumn{2}{|l|}{ Gender } \\
\hline Male & $24(63.2)$ \\
\hline Female & $14(36.8)$ \\
\hline \multicolumn{2}{|l|}{ Nationality } \\
\hline Saudi & $28(73.7)$ \\
\hline Non-Saudi & $10(26.3)$ \\
\hline \multicolumn{2}{|l|}{ Age } \\
\hline Median (IQR) & $66.5(54.3-77.5)$ \\
\hline \multicolumn{2}{|c|}{ Stroke severity scores at admission } \\
\hline \multicolumn{2}{|c|}{ NIHSS } \\
\hline Median (IQR) & $10.0(5.0-14.2)$ \\
\hline \multicolumn{2}{|l|}{ GCS } \\
\hline Median (IQR) & $14.5(13.0-15.0)$ \\
\hline \multicolumn{2}{|l|}{ Risk factors } \\
\hline HTN & $28(73.7)$ \\
\hline $\mathrm{DM}$ & $25(65.8)$ \\
\hline Hyperlipidemia & $24(63.2)$ \\
\hline $\mathrm{IHD}^{*}$ & $23(60.5)$ \\
\hline Previous stroke & $17(44.7)$ \\
\hline A-Fib & $14(36.8)$ \\
\hline Smoking & $12(31.6)$ \\
\hline $\mathrm{CHF}^{\dagger}$ & $5(13.2)$ \\
\hline Previous TIA & $3(7.9)$ \\
\hline Alcohol use & $1(2.6)$ \\
\hline \multicolumn{2}{|l|}{ Ischemic etiology } \\
\hline Cardio-embolic & $21(55.3)$ \\
\hline Large artery disease & $2(5.3)$ \\
\hline Small artery disease lacunar & $1(2.6)$ \\
\hline Other & $1(2.6)$ \\
\hline Unknown & $13(34.2)$ \\
\hline \multicolumn{2}{|c|}{ Medications prior to hospitalization } \\
\hline Antiplatelet & $19(50)$ \\
\hline Dual Antiplatelet & $18(47.4)$ \\
\hline Anticoagulation & $5(13.2)$ \\
\hline Lipid-lowering agents & $10(26.3)$ \\
\hline Antihypertensive drug & $26(86.4)$ \\
\hline Hypoglycemic drug & $16(42.1)$ \\
\hline $\begin{array}{l}\text { *IHD - Ischemic heart dis } \\
\text { failure, }{ }^{*} \text { TIA - Tra }\end{array}$ & $\begin{array}{l}\text { - Congestive heart } \\
\text { emic attack }\end{array}$ \\
\hline
\end{tabular}

(15.7\%) had posterior circulation. Only one patient (2.6\%) had both anterior and posterior (Table 3 ).

Nine patients $(23.7 \%)$ received tPA, and 6 patients (15.8\%) underwent endovascular therapy. Twenty-five patients $(65.8 \%)$ were admitted to the intensive care unit (ICU). A foley catheter was applied to 18 patients (47.4\%), and 5 patients (13.2\%) were intubated; 37 patients $(97.4 \%)$ received antiplatelets, and 34 patients $(89.5 \%)$ received deep venous thrombosis (DVT) prophylactic. Eleven patients $(28.9 \%)$ received anticoagulant, and 36 patients $(94.7 \%)$ received lipid lowering agents (Table 4).

Other neurological complications were reported as depression (18.4\%) and epileptic seizure (13.2\%); DVT developed in 2 patients (5.3\%). The median NIHSS score at discharge was $5(3-10.2)$. Five patients show 4 points drop in NIHSS score after HT. There were 11 patients (28.9\%) who required a hospital stay less than one week, 10 patients $(26.3 \%)$ were between 8 to 14 days, and 17 patients (44.7\%) were more than 2 weeks. Only one patient $(2.6 \%)$ died during hospitalization and accounted for $(2.6 \%)$ of the total HT in patients with ischemic stroke (Table 5).

Discussion. The HT is one of the major complications of AIS, which can severely affect the outcome. This retrospective study describes the incidence rate, risk factors, clinical presentation, and outcomes of HT in patients with AIS admitted to a tertiary hospital

Table 2 - Vital signs and lab findings of patients with HT at admission.

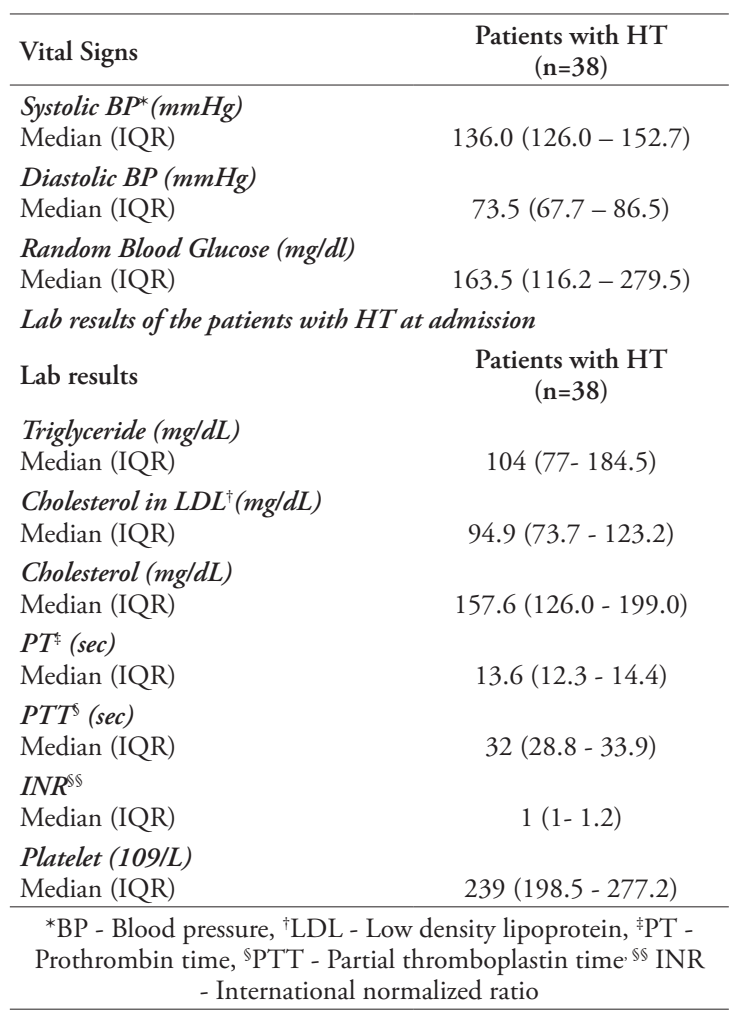


Table 3 - Radiological feature in patients with HT.

\begin{tabular}{lc}
\hline Variables & $\begin{array}{c}\text { Patients with HT } \\
(\mathbf{n}=38)\end{array}$ \\
\hline Type of HT & \\
Hemorrhagic infarction & $22(57.9)$ \\
Parenchymal hematoma & $16(42.1)$ \\
Infract size & \\
Small & $5(13.2)$ \\
Medium to large & $33(86.8)$ \\
Stroke distribution & \\
Anterior circulation & $31(81.5)$ \\
Posterior circulation & $6(15.7)$ \\
Both & $1(2.6)$ \\
\hline
\end{tabular}

Table 4 - Management parameters and prescribed medications for patients with HT.

\begin{tabular}{lc}
\hline Variables & $\begin{array}{c}\text { Patients with HT } \\
(\mathbf{n}=38) \mathbf{n}(\%)\end{array}$ \\
\hline Management parameters & \\
tPA & $9(23.7)$ \\
Endovascular therapy & $6(15.8)$ \\
ICU admission & $25(65.8)$ \\
Intubation & $5(13.2)$ \\
Foley catheter & $18(47.4)$ \\
Medication during hospitalization before HT & \\
Antiplatelet & $37(97.4)$ \\
DVT prophylactic & $34(89.5)$ \\
Anticoagulation & $11(28.9)$ \\
Lipid-lowering agents & $36(94.7)$ \\
Antihypertensive drug & $31(81.6)$ \\
Hypoglycemic drug & $18(47.7)$ \\
\hline
\end{tabular}

\section{Population}

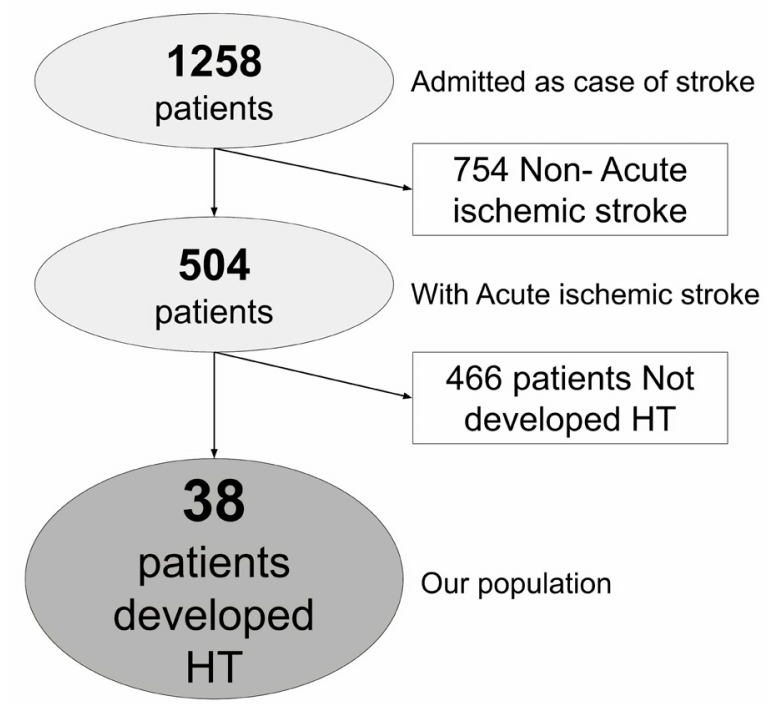

Figure 1 - Population.
Table 5 - Complications and outcomes data among HT.

\begin{tabular}{lc}
\hline Variables & $\begin{array}{c}\text { Patients with HT } \\
(\mathbf{n}=38)\end{array}$ \\
\hline Complications during hospitalization & $1(2.6)$ \\
Pulmonary embolism & $2(5.3)$ \\
Pneumonia & $5(13.2)$ \\
Epileptic seizure & $7(18.4)$ \\
Depression & $2(5.3)$ \\
DVT & \\
Stroke Severity scores at discharge & \\
NIHSS at discharge & $5(3-10.2)$ \\
Median (IQR) & \\
Length of stay & $11(28.9)$ \\
$<=1$ weeks & $10(26.3)$ \\
8-14 days & $17(40.7)$ \\
$>2$ weeks & \\
Outcome & $34(89.4)$ \\
Improved & $2(5.2)$ \\
Transferred to another facility & $1(2.6)$ \\
DAMA* & $1(2.6)$ \\
Death & $5(13.2)$ \\
Drop in NIHSS 4 points & *DAMA - Discharge against medical advice \\
\hline \multicolumn{2}{c}{} \\
\hline
\end{tabular}

in Kingdom of Saudi Arabia from August 2011 to April 2019. There were 504 patients with AIS: 321 males (63.69\%) and 183 females (36.31\%). Of those, HT was detected in 38 patients (8\%): 24 males (63.2\%) and 14 females $(36.8 \%)$. The incidence of HT is $(7.48 \%)$ in males and $(7.65 \%)$ in females in our sample. Studies show the incidence of HT is variable depending on many factors including the study design and inclusion criteria. The incidence of HT after r-tPA is $10-30 \%$. ${ }^{4}, 12$ However, the incidence rate is $(15 \%)$ in our population. Accordingly, most patients who received r-tPA therapy had a better prognosis compared to patients who did not receive r-tPA therapy. ${ }^{13,16}$ Some studies consider $\mathrm{r}-\mathrm{tPA}$ as a risk factor for PH. ${ }^{17,18}$

We found that HTN (73.7\%), DM (65.8\%), hyperlipidemia (63.2\%), and A-Fib (36.8\%) are the most common comorbidities. Several studies reported that DM and HTN are risk factors for HT. ${ }^{16,19}$ In our study, hyperlipidemia was seen in 24 patients (63.2\%). Other studies showed a relation between low LDL level and HT and explained this via the involvement of cholesterol in the integrity of blood vessel; therefore, low cholesterol levels lead to the weak blood vessel and subsequent rupture resulting in hemorrhage..$^{19,20}$ However other studies showed no association between the level of cholesterol and HT and mentioned that the relation between HT \& dyslipidemia remains controversial. 
We also found HT was encountered more in our sample with patients who were found to have large infarct size $(86.8 \%)$. This could be because cardiac embolism is accompanied by large vessel occlusion. This leads to a large infarct size which considered to be a risk for HT. ${ }^{19}$, ${ }^{21}$ In our study, half of the HT patients (47.4\%) were on dual antiplatelet therapy. We cannot determine if such treatment is a risk factor or not. In a match study which took 18 months, they found that patients with ischemic stroke who had both clopidogrel (Plavix) and aspirin together had an insignificant decrease in major vascular events, while they were found to have an increasing risk of life threatening events, and major bleeding. ${ }^{22}$

There are some limitations of our study: First, our study conducted in one center so our population may not be representative in large scale. Second, our sample is small, so it is hard to come to conclusion about the clinically significant of different variables. Thus, further large sample size, prospective studies of $\mathrm{HT}$ will provide more significant clinical information. Future research idea to take the HT patients and compare them with the non-HT patients across all the different variables.

In conclusion, old age, DM, hypertension, and hyperlipidemia are frequently encountered with stroke patients who developed HT. The HT was detected in 38 patients accounting for $8 \%$ of all AIS patients. Followup brain CT could be of value even if the patient is asymptomatic to identify the HT early to get a better outcome.

Acknowledgement. The authors would like to thank (www.americanmanuscripteditors.com) for the English language editing.

\section{References}

1.Sussman ES, Connolly ES, Jr. Hemorrhagic transformation: a review of the rate of hemorrhage in the major clinical trials of acute ischemic stroke. Front Neurol 2013; 4: 69.

2.Jickling GC, Manolescu BN. Breaking down barriers to identify hemorrhagic transformation in ischemic stroke. Neurology 2012; 79: 1632-1633.

3.Khatri P, Wechsler LR, Broderick JP. Intracranial hemorrhage associated with revascularization therapies. Stroke 2007; 38: 431-440.

4.Toni D, Fiorelli M, Bastianello S, Sacchetti ML, Sette G, Argentino C, et al. Hemorrhagic transformation of brain infarct: predictability in the first 5 hours from stroke onset and influence on clinical outcome. Neurology 1996; 46: 341-345.

5.Hornig CR, Dorndorf W, Agnoli AL. Hemorrhagic cerebral infarction--a prospective study. Stroke 1986; 17: 179-185.

6.Balami JS, Chen RL, Grunwald IQ, Buchan AM. Neurological complications of acute ischaemic stroke. Lancet Neurol 2011; 10: 357-371.
7.Marcell László J, Hortobágyi T. Hemorrhagic transformation of ischemic stroke. Vascular Diseases and Therapeutics 2017; 2.

8. Maeshima S, Okamoto S, Okazaki H, Mizuno S, Asano N, Tsunoda T, et al. Hemorrhagic Transformation in Patients with Cerebral Infarction Referred to a Rehabilitation Hospital. Interv Neurol 2016; 4: 69-74.

9.Kazmierski R, Michalak S, Wencel-Warot A, Nowinski WL. Serum tight-junction proteins predict hemorrhagic transformation in ischemic stroke patients. Neurology 2012; 79: $1677-85$.

10.Aviv RI, d'Esterre CD, Murphy BD, Hopyan JJ, Buck B, Mallia $\mathrm{G}$, et al. Hemorrhagic transformation of ischemic stroke: prediction with CT perfusion. Radiology 2009; 250: 867-877.

11.Kerenyi L, Kardos L, Szasz J, Szatmari S, Bereczki D, Hegedus $\mathrm{K}$, et al. Factors influencing hemorrhagic transformation in ischemic stroke: a clinicopathological comparison. Eur J Neurol 2006; 13: 1251-1255.

12.Lindley RI, Wardlaw JM, Sandercock PA, Rimdusid P, Lewis SC, Signorini DF, et al. Frequency and risk factors for spontaneous hemorrhagic transformation of cerebral infarction. I Stroke Cerebrovasc Dis 2004; 13: 235-246.

13.Ge WQ, Chen J, Pan H, Chen F, Zhou CY. Analysis of Risk Factors Increased Hemorrhagic Transformation after Acute Ischemic Stroke. J Stroke Cerebrovasc Dis 2018; 27: 3587-3590.

14.Fiorelli M, Bastianello S, von Kummer R, del Zoppo GJ, Larrue V, Lesaffre E, et al. Hemorrhagic transformation within 36 hours of a cerebral infarct: relationships with early clinical deterioration and 3-month outcome in the European Cooperative Acute Stroke Study I (ECASS I) cohort. Stroke 1999; 30: 2280-2284.

15.Fisher CM. Lacunar strokes and infarcts: a review. Neurology 1982; 32: 871-876.

16.Moriya Y, Takahashi W, Kijima C, Yutani S, Iijima E, Mizuma A, et al. Predictors for hemorrhagic transformation with intravenous tissue plasminogen activator in acute ischemic stroke. Tokai J Exp Clin Med 2013; 38: 24-27.

17.Paciaroni M, Agnelli G, Corea F, Ageno W, Alberti A, Lanari A, et al. Early hemorrhagic transformation of brain infarction: rate, predictive factors, and influence on clinical outcome: results of a prospective multicenter study. Stroke 2008; 39: 2249-2256.

18.Larrue V, von Kummer R, del Zoppo G, Bluhmki E. Hemorrhagic transformation in acute ischemic stroke. Potential contributing factors in the European Cooperative Acute Stroke Study. Stroke 1997; 28: 957-960.

19.Terruso V, D’Amelio M, Di Benedetto N, Lupo I, Saia V, Famoso G, et al. Frequency and determinants for hemorrhagic transformation of cerebral infarction. Neuroepidemiology 2009; 33: 261-265.

20.D'Amelio M, Terruso V, Famoso G, Ragonese P, Aridon P, Savettieri G. Cholesterol levels and risk of hemorrhagic transformation after acute ischemic stroke. Cerebrovasc Dis 2011; 32: 234-238.

21.Valentino F, Gentile L, Terruso V, Mastrilli S, Aridon P, Ragonese P, et al. Frequency and determinants for hemorrhagic transformation of posterior cerebral stroke: Posterior ischemic stroke and hemorrhagic transformation. BMC Res Notes 2017; 10: 592.

22.Zhao R, Feng XY, Zhang M, Shen XL, Su JJ, Liu JR. Progressive hemorrhagic transformation following dual antiplatelet therapy. CNS Neurosci Ther 2014; 20: 92-94. 